Erratum

\title{
Erratum to "Reinstitution of Mechanical Ventilation within 14 Days as a Poor Predictor in Prolonged Mechanical Ventilation Patients following Successful Weaning"
}

\author{
Mei-Lien Tu, ${ }^{1,2}$ Ching-Wan Tseng, ${ }^{1,2}$ Yuh Chyn Tsai, ${ }^{1,2}$ Chin-Chou Wang, ${ }^{1,3}$ Chia-Cheng \\ Tseng, ${ }^{3}$ Meng-Chih Lin, ${ }^{1,3}$ Wen-Feng Fang, ${ }^{1,3}$ Yung-Che Chen, ${ }^{3}$ and Shih-Feng Liu ${ }^{1,3}$ \\ ${ }^{1}$ Department of Respiratory Therapy, Kaohsiung Chang Gung Memorial Hospital, \\ Chang Gung University College of Medicine, Kaohsiung, Taiwan \\ ${ }^{2}$ Chang Gung University of Science and Technology, Taiwan \\ ${ }^{3}$ Division of Pulmonary \& Critical Care Medicine, Department of Internal Medicine, Kaohsiung Chang Gung Memorial Hospital, \\ Chang Gung University College of Medicine, 123, Ta-Pei Road, Niao-Sung Hsiang, Kaohsiung Hsien, Taiwan
}

Correspondence should be addressed to Shih-Feng Liu; m85d@adm.cgmh.org.tw

Received 1 July 2013; Accepted 2 August 2013

Copyright (C) 2013 Mei-Lien Tu et al. This is an open access article distributed under the Creative Commons Attribution License, which permits unrestricted use, distribution, and reproduction in any medium, provided the original work is properly cited.

In the paper titled "Reinstitution of Mechanical Ventilation within 14 Days as a Poor Predictor in Prolonged Mechanical Ventilation Patients following Successful Weaning," the affiliations of the authors should be corrected as shown above. 


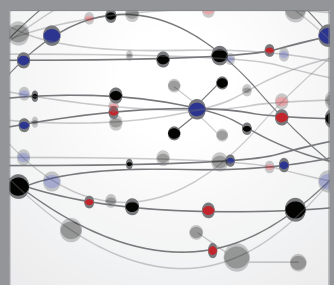

The Scientific World Journal
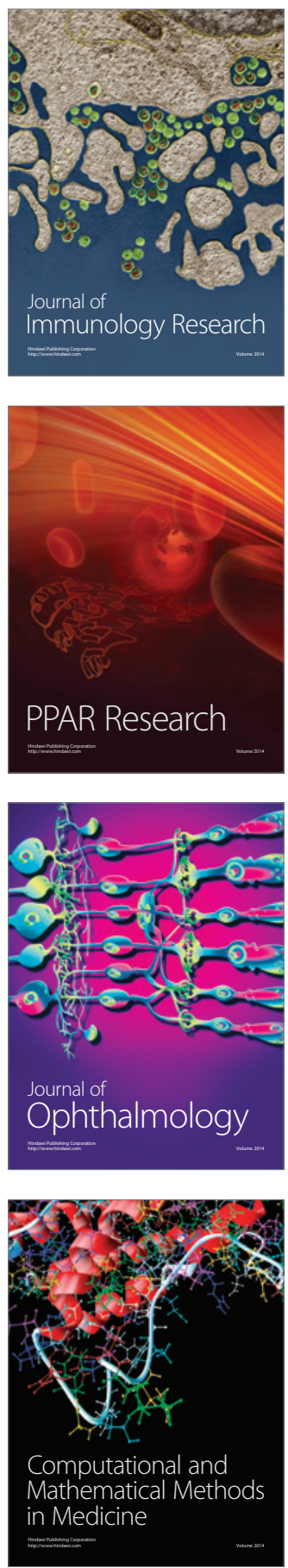

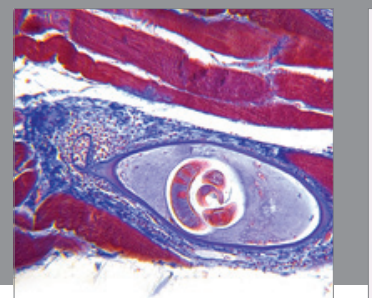

Gastroenterology

Research and Practice
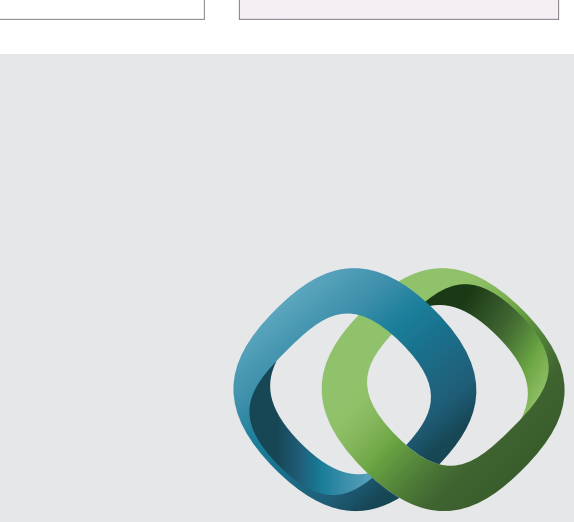

\section{Hindawi}

Submit your manuscripts at

http://www.hindawi.com
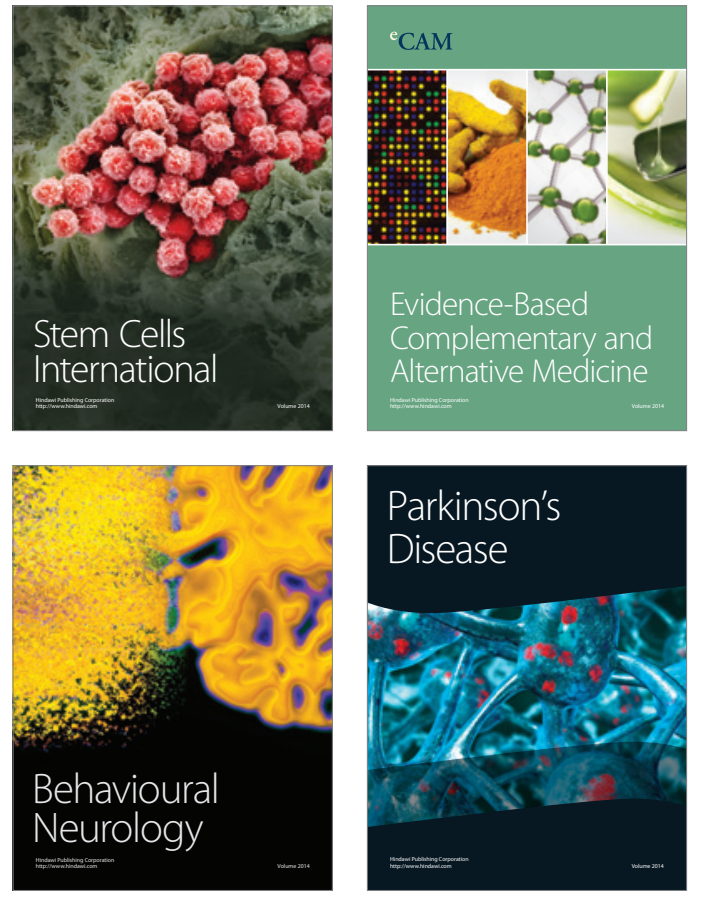
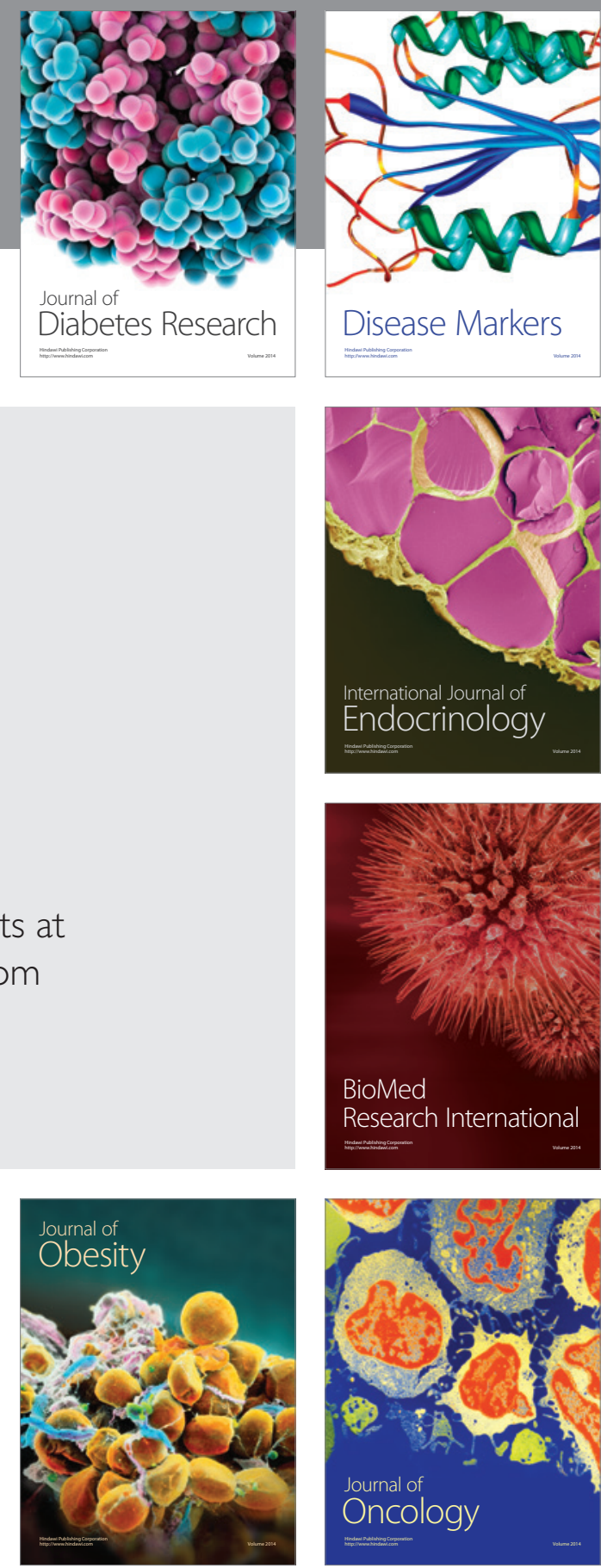

Disease Markers
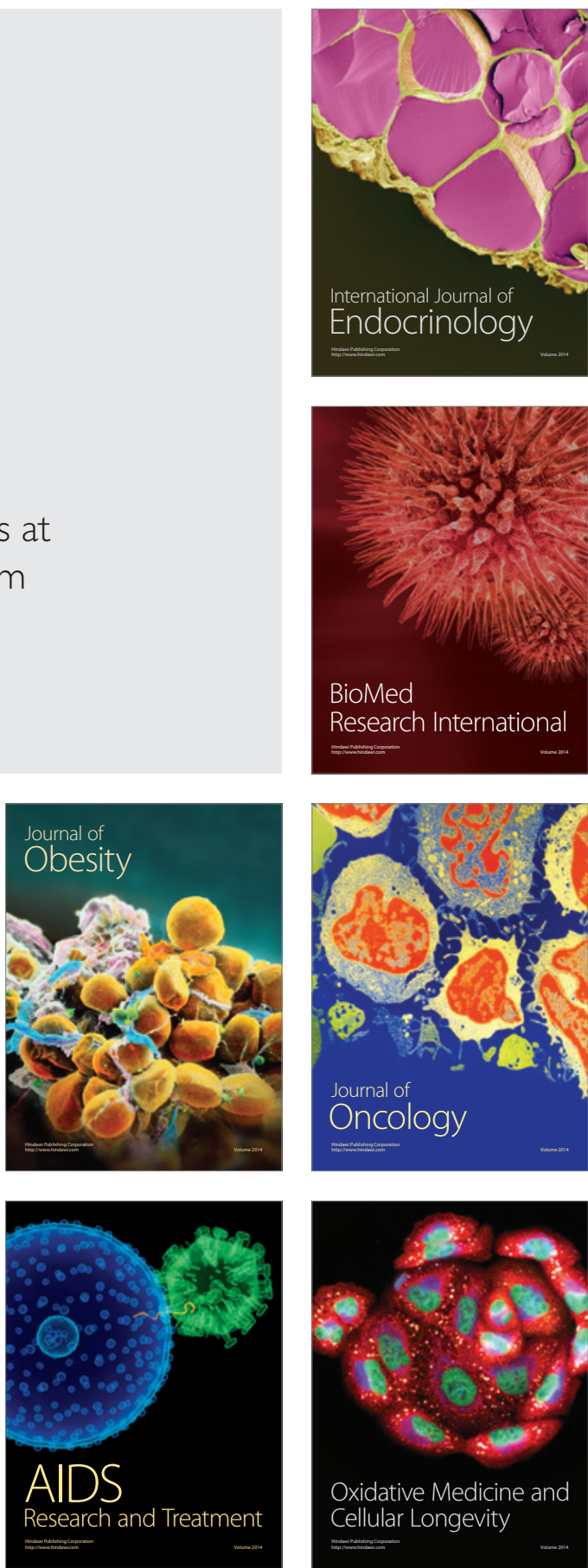